\title{
Are targeted agents the key to unlock the code for immune checkpoint inhibitors in soft-tissue sarcomas?
}

\author{
Tarek Assi*,1 \\ ${ }^{1}$ Hematology-Oncology Department, Faculty of Medicine, Saint-Joseph University, Beirut, Lebanon \\ *Author for correspondence: Tel.: 009613925554; Tarekassi@gmail.com
}

\begin{abstract}
"With the limited benefit of cytotoxic chemotherapy, targeted therapy and ICls alone in STS patients, huge efforts are being invested to assess the efficacy of drug combinations in these patients"
\end{abstract}

First draft submitted: 18 May 2019; Accepted for publication: 4 July 2019; Published online: 3 October 2019

Soft-tissue sarcomas (STS) are a heterogeneous group of malignant mesenchymal tumors accounting for only $1 \%$ of all cancers [1]. With more than 70 distinct histological subtypes, up to 12,000 STS patients are diagnosed yearly in the USA with approximately 5000 deaths [2]. Despite all the progress in the cancer field, the prognosis of STS patients remains dismal with a mere overall survival (OS) of 12-14 months with cytotoxic chemotherapy [3]. Recently, the oncology field have been subject to a paradigm shift in the management of a multitude of solid tumors with the arrival of immune checkpoint inhibitors (ICIs) [4-6]. The main mechanism of action of these novel agents is to restore the activity of the immune system from its hibernating state through interaction with immune checkpoints (PD-L1 and PD-1), mainly expressed on tumor cells, tumor-infiltrating lymphocytes and antigen-presenting cells $[7,8]$. With the limited benefit of cytotoxic chemotherapy, targeted therapy and ICIs alone in STS patients, huge efforts are being invested to assess the efficacy of drug combinations in these patients.

The immune profile of STS as well as potential biomarkers of response to targeted therapy and ICIs are not yet well elucidated. In a large analysis of 2000 STSs, half demonstrated positive expression of PD-L1 with the highest levels in undifferentiated pleomorphic sarcoma, leiomyosarcoma, liposarcoma and chondrosarcomas [9]. In fact, PD-L1 expression in STS have different levels of expression ranging from 12 to $65 \%$ on tumor cells and lymphocytes [10]. Nevertheless, there are discordant results concerning a significant correlation between clinical presentation, overall survival and PD-L1 expression [10,11]. Also, after evaluation of more than 750 genes using the Nanostring platform, a higher frequency of antigen presentation and T-cell infiltration was noted in the most common STS subtypes including undifferentiated pleomorphic sarcoma and LMS patients [12]. High mutational and neoantigen burden (MB) were associated with improved oncological outcomes in patients receiving ICIs [13-15]. On average, STS are considered to have an intermediate MB in comparison to other tumors with high MB such as melanoma [16]. On the other hand, recent reports have demonstrated that VEGF, an angiogenesis promoter, can induce an immunosuppressive response within the tumor microenvironment by direct inhibition of T-cell function, activation and differentiation of dendritic cells as well as accumulation of myeloid-derived suppressor cells and regulatory $\mathrm{T}$ cells [17]. All that said, there is a solid rationale in the association of ICIs with anti-angiogenic agents in order to enhance the immune response in the tumor microenvironment.

First, the introduction of ICIs as single agents in STS has failed to exhibit a substantial impact on oncological outcomes. Retrospective and small prospective trials did not induce significant responses in different histological subtypes $[18,19]$. Two Phase II trials enrolling different categories of STSs confirmed the limited role of ICIs as single agents. In the first study, the Alliance 091401 study, a Phase II randomized noncomparative study, 85 patients with locally advanced (LA) or metastatic STS were randomized to receive nivolumab alone ( 42 patients) or in association with ipilimumab for four doses (43 patients). Overall response rate (ORR) with nivolumab was 5\% while it reached $16 \%$ in the combination arm. Median progression-free survival (PFS) was 1.7 and 4.1 months, respectively, while the overall survival (OS) were 10.7 and 14.3 months in the monotherapy and combination group, respectively [20].

Future Medicine 
The second trial was the SARCO28, a multicentric open-label Phase II trial with two cohorts of 40 patients, each evaluating the activity of pembrolizumab as monotherapy (STSs in the first cohort and bone tumors in the second). Also, the outcomes were limited in STS patients with an ORR of $18 \%$ and a median PFS and OS of 18 and 49 weeks, respectively [21].

Second, the addition of cytotoxic chemotherapy to ICIs did not improve the awaited results. For instance, a Phase II multicentric trial assessing the association of pembrolizumab with metronomic cyclophosphamide in 57 STS patients had only one significant response (solitary fibrous tumor) and a median PFS of only 1.4 months in all patient subgroups [22]. Chawla et al. analyzed retrospectively the combination of trabectedin, an alkylating agent approved in the second-line therapy of metastatic STS, to nivolumab in 28 STS patients with an ORR of $18.2 \%$, a median PFS of $>45.5$ weeks and a median OS of 91 weeks [23]. However, the first successful data were reported with the IMMUNOSARC trial, a Phase I/II trial conducted to evaluate the synergistic activity of nivolumab and sunitinib, anti-VEGF inhibitor, in STS patients. According to the RECIST criteria, 6 out of 14 evaluable patients had at least a partial response (42.8\%) thus encouraging the possible synergistic activity of anti-angiogenic agents with ICIs [19].

Recently, a Phase II single-center nonrandomized trial evaluated the role of axitinib, $(5 \mathrm{mg}$ twice daily then escalating dose to $10 \mathrm{mg}$ twice daily) in combination with pembrolizumab (200 $\mathrm{mg}$ flat dose) every 3 weeks in LA advanced or metastatic STS patients. Approximately 33 patients were enrolled including 12 patients with alveolar soft part sarcoma (ASPS; 36\%). Interestingly, the 3-month PFS, the primary end point of this trial, was 65.6\% in the overall population and $72.7 \%$ in ASPS patients (the median PFS was 4.7 months). Median OS for the whole population was 18.7 months with 1 -year OS of $72 \%$. Also, a high response rate of $25 \%$ was noted with clinical benefit rate of $53.1 \%$. The association was tolerated with $39 \%$ grade $3-4$ side effects. The most common side effects were hypertension (15\%) and auto-immune toxicities (15\%) while $21 \%$ of patients exhibiting serious side effects [24]. Even though the tumor cell PD-L1 was expressed in 52\% of evaluable patients and all ASPS patients, neither PD-L1 expression nor the tumor infiltrating score did correlate with the oncological outcomes. Exciting findings from this study is that patients with high neutrophil-to-lymphocyte ratio and high plasma baseline angiogenic activity were associated with increased response to ICIs and axitinib [24]. On the other hand, ASPS, a rare subtype of STS characterized by an unbalanced translocation $\mathrm{t}(\mathrm{X} ; 17)$, have demonstrated increased sensitivity to tyrosine kinase inhibitors such as cediranib, pazopanib or sunitinib [25]. Also, Atezolizumab, an anti-PD-L1 agent, successfully induced significant responses in $8(42 \%)$ out of 19 ASPS patients [26]. The association of axitinib and pembrolizumab demonstrated an enhanced synergism for both agents in ASPS patients with an ORR in ASPS patients being 54.5\% with median PFS 12.4 months in ASPS. These results constitute a solid ground for successful drug combinations with higher response rates in comparison to ICIs or targeted agents alone in all the STS histological subtypes, more particularly in ASPS patients.

Until recently, ICIs failed to earn a substantial role in STS with limited impact on response rates and survival. The combination of targeted agents to ICIs have changed the current reigning paradigm in some solid tumors with the recent FDA approvals of axitinib in combination pembrolizumab or avelumab in the first-line therapy of metastatic renal cell carcinoma [27,28]. These exhilarating signs of synergism and activity in several STS subtypes between targeted agents and ICIs should encourage sarcoma enthusiasts into conducting larger trials in order to identify validated biomarkers and address the unmet need of optimizing the therapeutic arsenal against advanced STS.

Financial \& competing interests disclosure

The authors have no relevant affiliations or financial involvement with any organization or entity with a financial interest in or financial conflict with the subject matter or materials discussed in the manuscript. This includes employment, consultancies, honoraria, stock ownership or options, expert testimony, grants or patents received or pending, or royalties.

No writing assistance was utilized in the production of this manuscript.

\section{References}

1. Siegel R, Ma J, Zou Z, Jemal A. Cancer statistics, 2014. CA: Cancer J. Clinicians 64(1), 9-29 (2014).

2. Siegel RL, Miller KD, Jemal A. Cancer statistics, 2015. CA: Cancer J. Clinicians 65(1), 5-29 (2015).

3. Italiano A, Mathoulin-Pelissier S, Le Cesne A, et al. Trends in survival for patients with metastatic soft-tissue sarcoma. Cancer 117(5), 1049-1054 (2011). 
4. Borghaei H, Paz-Ares L, Horn L et al. Nivolumab versus docetaxel in advanced nonsquamous non-small-cell lung cancer. N. Engl. J. Med. 373(17), 1627-1639 (2015).

5. Larkin J, Chiarion-Sileni V, Gonzalez R et al. Combined nivolumab and ipilimumab or monotherapy in untreated melanoma. $N$. Engl. J. Med. 373(1), 23-34 (2015).

6. Motzer RJ, Escudier B, McDermott DF et al. Nivolumab versus everolimus in advanced renal-cell carcinoma. N. Engl. J. Med. 373(19), 1803-1813 (2015).

7. Miwa S, Nishida H, Tsuchiya H. Current status of immunotherapy for sarcomas. Immunotherapy 9(16), 1331-1338 (2017).

8. Park J-Y, Jin KL, Kim D-Y et al. Surgical staging and adjuvant chemotherapy in the management of patients with adult granulosa cell tumors of the ovary. Gynecol. Oncol. 125(1), 80-86 (2012).

9. Movva S, Wen W, Chen W et al. Multi-platform profiling of over 2000 sarcomas: identification of biomarkers and novel therapeutic targets. Oncotarget 6(14), 12234 (2015).

10. Kim JR, Moon YJ, Kwon KS et al. Tumor infiltrating PD1-positive lymphocytes and the expression of PD-L1 predict poor prognosis of soft-tissue sarcomas. PloS ONE 8(12), e82870 (2013).

11. D'Angelo SP, Shoushtari AN, Agaram NP et al. Prevalence of tumor-infiltrating lymphocytes and PD-L1 expression in the soft-tissue sarcoma microenvironment. Hum. Pathol. 46(3), 357-365 (2015).

12. Pollack SM, He Q, Yearley JH et al. T-cell infiltration and clonality correlate with programmed cell death protein 1 and programmed death-ligand 1 expression in patients with soft-tissue sarcomas. Cancer 123(17), 3291-3304 (2017).

13. Johnson DB, Frampton GM, Rioth MJ et al. Hybrid capture-based next-generation sequencing (HC NGS) in melanoma to identify markers of response to anti-PD-1/PD-L1. Am. Soc. Clin. Oncol. 34, 105 (2016).

14. Rizvi NA, Hellmann MD, Snyder A et al. Mutational landscape determines sensitivity to PD-1 blockade in non-small cell lung cancer. Science 348(6230), 124-128 (2015).

15. Gibney GT, Weiner LM, Atkins MB. Predictive biomarkers for checkpoint inhibitor-based immunotherapy. Lancet Oncol. 17(12), e542-e551 (2016).

16. Veenstra R, Kostine M, Cleton-Jansen A-M, de Miranda NF, Bovée JV. Immune checkpoint inhibitors in sarcomas: in quest of predictive biomarkers. Lab. Investigat. 98(1), 41 (2018).

17. Yang J, Yan J, Liu B. Targeting VEGF/VEGFR to modulate antitumor immunity. Front. Immunol. 9, 9 (2018).

18. Ben-Ami E, Barysauskas CM, Solomon $S$ et al. Immunotherapy with single agent nivolumab for advanced leiomyosarcoma of the uterus: results of a Phase II study. Cancer 123(17), 3285-3290 (2017).

19. Martin Broto J, Hindi N, Redondo A et al. IMMUNOSARC: a collaborative Spanish (GEIS) and Italian (ISG) Sarcoma Groups Phase I/II trial of sunitinib plus nivolumab in selected bone and soft-tissue sarcoma subtypes - Results of the Phase I part. Am. Soc. Clin. Oncol.36, 11515 (2018).

20. D'Angelo SP, Mahoney MR, Van Tine BA et al. Nivolumab with or without ipilimumab treatment for metastatic sarcoma (Alliance A091401): two open-label, non-comparative, randomised, Phase II trials. Lancet Oncol. 19(3), 416-426 (2018).

21. Mir O, Honoré C, Adam J. PD-1 inhibition in bone sarcoma and soft-tissue sarcoma. Lancet Oncol. 18(11), 1430-1431 (2017).

22. Toulmonde M, Penel N, Adam J et al. Use of PD-1 targeting, macrophage infiltration, and IDO pathway activation in sarcomas: a Phase II clinical trial. JAMA Oncol. 1, 4(1), 93-7 janv (2018).

23. Chawla SP, Sankhala KK, Ravicz J et al. Clinical experience with combination chemo-/immunotherapy using trabectedin and nivolumab for advanced soft-tissue sarcoma. Am. Soc. Clin. Oncol. 36, (2018).

24. Wilky BA, Trucco MM, Subhawong TK et al. Axitinib plus pembrolizumab in patients with advanced sarcomas including alveolar soft-part sarcoma: a single-centre, single-arm, Phase II trial. Lancet Oncol. 20, 837-848 (2019).

25. Paoluzzi L, Maki RG. Diagnosis, prognosis, and treatment of alveolar soft-part sarcoma: a review. JAMA oncol. 5(2), 254-260 (2019).

26. O'Sullivan Coyne G, Sharon E, Moore N. Phase II study of atezolizumab in patients with alveolar soft part sarcoma. Connective Tissue Oncology Society Annual Meeting, Rome, Italy, November 14-17 (2018).

27. Motzer RJ, Penkov K, Haanen J et al. Avelumab plus axitinib versus sunitinib for advanced renal-cell carcinoma. N. Engl. J. Med. 380(12), 1103-1115 (2019).

28. Rini BI, Plimack ER, Stus V et al. Pembrolizumab plus axitinib versus sunitinib for advanced renal-cell carcinoma. N. Engl. J. Med. 380(12), 1116-1127 (2019). 
